Homology, Homotopy and Applications, vol.15(2), 2013, pp.127-144

\title{
EFFECTIVE DESCENT MORPHISMS IN STAR-REGULAR CATEGORIES
}

\author{
MARINO GRAN AND OLIVETTE NGAHA NGAHA
}

\author{
(communicated by George Janelidze)
}

\begin{abstract}
In this article a sufficient condition on a star-regular category is introduced guaranteeing that regular epimorphisms are effective descent morphisms. This condition is satisfied by any category with a good theory of ideals (thus, in particular, by any ideal determined category), by any almost abelian category (for instance, by the categories of torsion abelian groups, torsion-free abelian groups, normed vector spaces, Banach spaces, locally compact abelian groups, etc.) and by any category of topological Mal'tsev algebras (in particular, by the category of topological groups).
\end{abstract}

\section{Introduction}

A finitely complete category $\mathbb{C}$ is regular when

(a) Any arrow $f: A \rightarrow B$ in $\mathbb{C}$ can be factorised as $f=i q$

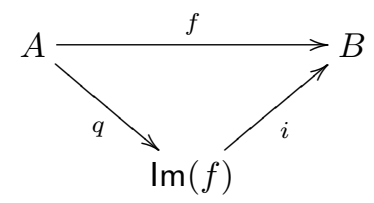

with $q$ a regular epimorphism and $i$ a monomorphism;

(b) These factorisations are pullback-stable in $\mathbb{C}$.

In the category of sets, and, more generally, in any variety of universal algebras, an arrow is a regular epimorphism precisely when it is surjective. Accordingly, the factorisation in (a) is the usual one of a function (or a homomorphism) as a surjection $q: A \rightarrow \operatorname{Im}(f)$ onto its direct image $\operatorname{Im}(f)=\{f(a) \mid a \in A\}$ followed by the inclusion $i: \operatorname{Im}(f) \rightarrow B$ of its image in its codomain $B$. Furthermore, surjective functions (and surjective homomorphisms) are clearly pullback-stable, so that these categories are

The second author's research has been supported by a Bourse de coopération au développement de l'Université catholique de Louvain.

Received November 30, 2012, revised January 20, 2013; published on November 16, 2013.

2000 Mathematics Subject Classification: 18A20, 18A30,18C99, 18 E05.

Key words and phrases: effective descent morphism, star-regular category, ideal of morphisms, semieffective star-regular category, almost abelian category, torsion theory, topological Mal'tsev algebra. Article available at http://intlpress.com/HHA/v15/n2/a7 and doi:10.4310/HHA.2013.v15.n2.a7 Copyright (C) 2013, International Press. Permission to copy for private use granted. 
all regular. For many purposes the notion of regular epimorphism provides a very suitable abstraction of the notion of quotient in the context of regular categories.

However, although regular epimorphisms are known to be descent morphisms in any regular category, they fail to be effective descent morphisms, in general [18]. This is due to the fact that not all equivalence relations in a regular category $\mathbb{C}$ are effective, namely they do not necessarily occur as the kernel pair of a morphism in $\mathbb{C}$. The recent work on descent theory (see [17], for instance, and the references therein) has made it clear that the right notion of "good quotient" in a general category is precisely provided by the notion of effective descent morphism.

The present paper deals with the problem of finding a simple condition guaranteeing that regular epimorphisms and effective descent morphisms coincide in a regular category. This is obviously the case when the regular category is exact [1] (as the category of sets or any variety of algebras is, for instance), since all equivalence relations are effective. However, this is also the case for many (not necessarily exact) regular categories: G. Janelidze and M. Sobral call such categories almost exact $[\mathbf{1 6}]$. The condition we propose in this article is expressed in the realm of star-regular categories recently introduced in $[\mathbf{1 0}]$, which are a special kind of regular categories equipped with an ideal of morphisms (in the sense of [7]), a concept that we recall in Section 2. We call semi-effective the star-regular categories satisfying this suitable condition (see Definition 2.11) which guarantees, under a mild further condition, that regular epimorphisms are effective descent morphisms (Theorem 3.2). Several interesting categories turn out to have this property, many of which are not exact: any "efficiently regular category" in the sense of D. Bourn [4], any "almost abelian" category in the sense of W. Rump [24] (see also [22]), and any "category with a good theory of ideals" in the sense of Z. Janelidze, A. Ursini and the first author [10]. In the last part of our work we analyse several further examples, some of which have been studied in the interesting article [8] by T. Everaert. A difference with that article is that here we adopt the "elementary approach" to descent theory (recalled here below) instead of the "monadic approach" used in [8].

\section{Acknowledgements}

The authors are grateful to Diana Rodelo, to Tomas Everaert and to Zurab Janelidze for many useful comments and suggestions on the subject of this article.

\section{Elementary descent theory}

Let $\mathbb{C}$ be a category with pullbacks. For a morphism $p: E \rightarrow B$ in $\mathbb{C}$, we denote by

$$
E q(p) \stackrel{p_{1}}{\underset{p_{2}}{\longrightarrow}} E
$$

the equivalence relation in $\mathbb{C}$ determined by the kernel pair of $p$. Given such an equivalence relation in $\mathbb{C}$, we write $\operatorname{DiscFib}(E q(p))$ for the category of discrete fibrations of equivalence relations over $E q(p)$ and natural morphisms: recall that an object in this 
category is an internal functor $\left(\varphi_{0}, \varphi_{1}\right): R \rightarrow E q(p)$ as in the commutative diagram

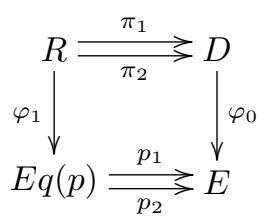

having the property that the square involving the second projections is a pullback (this implies that the square involving the first projections is a pullback). Let

$$
F^{p}:(\mathbb{C} \downarrow B) \rightarrow \operatorname{DiscFib}(E q(p))
$$

be the functor sending an object $(A, \alpha)$ in the comma category $(\mathbb{C} \downarrow B)$ to the discrete fibration $(C)$ in the commutative diagram

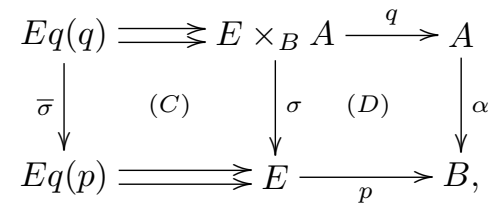

where $(D)$ is the pullback of $p$ and $\alpha, E q(q)$ is the kernel pair of $q$, and $\bar{\sigma}$ the induced arrow making the two left squares commutative.

A morphism $p: E \rightarrow B$ in $\mathbb{C}$ is said to be:

(a) a descent morphism if the functor $F^{p}$ is full and faithful,

(b) an effective descent morphism if the functor $F^{p}$ is a category equivalence.

Remark that a morphism $p: E \rightarrow B$ is an effective descent morphism as defined above exactly when the change-of-base functor $p^{*}:(\mathbb{C} \downarrow B) \rightarrow(\mathbb{C} \downarrow E)$ is monadic, as explained in $[\mathbf{1 7}]$, for instance. The following theorem will also be needed (see $[\mathbf{8}$, $16,17])$.

Theorem 1.1. Let $\mathbb{C}$ be a finitely complete regular category. Then

(1) $p: E \rightarrow B$ in $\mathbb{C}$ is a descent morphism if and only if it is a regular epimorphism;

(2) A regular epimorphism $p: E \rightarrow B$ in $\mathbb{C}$ is an effective descent morphism if and only if for any discrete fibration as in (1) over the kernel pair $E q(p)$ of $p$, the equivalence relation $R$ is effective.

\section{Star-regular categories}

In this section we recall some basic aspects of the theory of "star relations" in a regular "multi-pointed category", as introduced by Z. Janelidze, A. Ursini and the first author. We refer the reader to [10] for further details.

Definition 2.1. Let $\mathbb{C}$ be a category, $\mathcal{N}$ a class of morphisms of $\mathbb{C}$ that forms an ideal in the sense of C. Ehresmann [7]: for any composable pair of morphisms $f, g$ of $\mathbb{C}$ the composite $g f$ belongs to $\mathcal{N}$ whenever either $f$ or $g$ belongs to $\mathcal{N}$. A category $\mathbb{C}$ equipped with an ideal $\mathcal{N}$ of morphisms is called a multi-pointed category $[\mathbf{1 0}]$.

The following examples will be the guiding ones in this article: 
Example 2.2.

(1) Any category $\mathbb{C}$ can be seen as a multi-pointed category by choosing for $\mathcal{N}$ the class of all morphisms in $\mathbb{C}$ : this situation is usually referred to as the total context.

(2) Any pointed category, with zero object 0 , can be thought of as a multi-pointed category by choosing for $\mathcal{N}$ the class of zero morphisms: this is the pointed context.

Convention. From now on we shall assume that the category $\mathbb{C}$ is finitely complete.

A pair of parallel morphisms $\sigma=\left(\sigma_{1}, \sigma_{2}\right): S \rightrightarrows X$ is called a star when $\sigma_{1} \in \mathcal{N}$; it is called a monic star when the pair $\left(\sigma_{1}, \sigma_{2}\right)$ is jointly monomorphic. A morphism $k: K \rightarrow X$ is the $\mathcal{N}$-kernel of a morphism $f: X \rightarrow Y$ if the composite $f k$ belongs to $\mathcal{N}$ and, for any other morphism $g: L \rightarrow X$ such that $f g \in \mathcal{N}$, there exists a unique morphism $u: L \rightarrow K$ such that $k u=g$ :

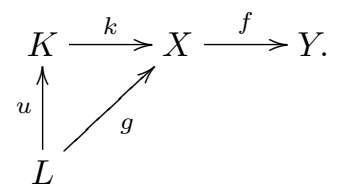

Note that such a $k$ is always a monomorphism. In the pointed context, the $\mathcal{N}$-kernel of a morphism $f: X \rightarrow Y$ is the classical kernel of this morphism; in the total context, the $\mathcal{N}$-kernel of $f: X \rightarrow Y$ is simply the identity morphism $1_{X}$.

For a relation $\varrho=\left(\varrho_{1}, \varrho_{2}\right): R \rightrightarrows X$ on an object $X$, we denote by $\varrho^{*}$ the largest subrelation of $\varrho$ which is a monic star. Such a star exists whenever the $\mathcal{N}$-kernels exist, since in this case it is given by $\varrho^{*}=\left(\varrho_{1} k, \varrho_{2} k\right): K \rightrightarrows X$, where $k: K \rightarrow R$ is the $\mathcal{N}$-kernel of $\varrho_{1}$. In particular, if $\Delta_{X}: X \rightrightarrows X$ is the discrete equivalence relation on $X$, we have $\Delta_{X}^{*}=\left(k_{X}, k_{X}\right): K \rightrightarrows X$, where $k_{X}: K \rightarrow X$, is the $\mathcal{N}$-kernel of $1_{X}$.

A kernel star (or a star-kernel) of a morphism $f: X \rightarrow Y$ is a star $\kappa=\left(\kappa_{1}, \kappa_{2}\right)$ : $K \rightrightarrows X$ such that $f \kappa_{1}=f \kappa_{2}$ and, for any other star $\kappa^{\prime}=\left(\kappa_{1}^{\prime}, \kappa_{2}^{\prime}\right): K^{\prime} \rightrightarrows X$ such that $f \kappa_{1}^{\prime}=f \kappa_{2}^{\prime}$, there exists a unique morphism $u: K^{\prime} \rightarrow K$ such that $\kappa u=\kappa^{\prime}$ :

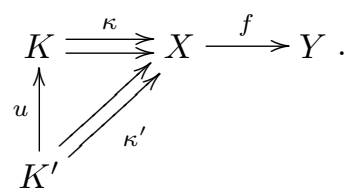

It is easy to see that, in the presence of $\mathcal{N}$-kernels, the kernel star of an arrow $f: X \rightarrow Y$ is given by $E q(f)^{*} \rightrightarrows X$.

In the pointed context, the notion of a kernel star of a morphism becomes the classical notion of a kernel of a morphism, while in the total context it gives the notion of a kernel pair of a morphism. Let us then consider a commutative diagram

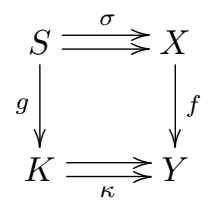


of stars and morphisms: $f \sigma=\kappa g$ means that $f \sigma_{1}=\kappa_{1} g$ and $f \sigma_{2}=\kappa_{2} g$. Such a diagram is called a star-pullback when, given a star $\sigma^{\prime}: S^{\prime} \rightrightarrows X$ and a morphism $g^{\prime}: S^{\prime} \rightarrow K$ such that $f \sigma^{\prime}=\kappa g^{\prime}$, there exists a unique morphism $h: S^{\prime} \rightarrow S$ such that $\sigma h=\sigma^{\prime}$ and $g h=g^{\prime}$.

Definition $2.3([\mathbf{1 0}])$. A star-regular category is a regular multi-pointed category $(\mathbb{C}, \mathcal{N})$ with $\mathcal{N}$-kernels in which every regular epimorphism is a coequaliser of a star.

In the total context, a star-regular category is simply a regular category. In the pointed context, a star-regular category is the same as a normal category in the sense of [19], i.e., a regular category in which any regular epimorphism is a normal epimorphism.

The following lemma provides a characterisation of those arrows that are monomorphisms in terms of their kernel star:

Lemma 2.4. In a star-regular category $\mathbb{C}$, the following conditions are equivalent for a morphism $f: X \rightarrow Y$ :

(1) $f: X \rightarrow Y$ is a monomorphism;

(2) $E q(f)^{*}=\Delta_{X}^{*}$;

(3) The projections $p_{1}: E q(f)^{*} \rightarrow X$ and $p_{2}: E q(f)^{*} \rightarrow X$ are equal.

Proof. It is obvious that that (1) implies (2), and let us prove that $(2) \Rightarrow(1)$. For this, we observe that the correspondence $\phi$ : KernelPairs $\longrightarrow$ KernelStars mapping any kernel pair $E q(f)$ in a star-regular category $\mathbb{C}$ to its corresponding star $E q(f)^{*}$ is a bijection, since any regular epimorphism in $\mathbb{C}$ is the coequaliser of its kernel star. Accordingly, $E q(f)^{*}=\Delta_{X}^{*}$ implies that $E q(f)=\Delta_{X}$, and $f: X \rightarrow Y$ is then a monomorphism. Finally, the implication $(2) \Rightarrow(3)$ is clear, whereas $(3) \Rightarrow(2)$ easily follows from the universal properties of the kernel stars $E q(f)^{*}$ and $\Delta_{X}^{*}$.

In the total context, Lemma 2.4 says, in particular, that $f$ is a monomorphism if and only if $E q(f)=\Delta_{X}$; in the pointed context, it says that $f$ is a monomorphism if and only if its kernel $\operatorname{ker}(f)$ is trivial: $\operatorname{ker}(f)=0$ (see [5] for the case of normal categories).

Corollary 2.5. A span $Y \stackrel{f}{<} X \stackrel{g}{\longrightarrow} Z$ in a star-regular category $\mathbb{C}$ is a relation if and only if $E q(f)^{*} \wedge E q(g)^{*}=\Delta_{X}^{*}$.

Proof. This follows from Lemma 2.4 and the fact that "starring" preserves meets, i.e., $E q(f)^{*} \wedge E q(g)^{*}=(E q(f) \wedge E q(g))^{*}$ (see Lemma 2.6 in $\left.[\mathbf{1 0}]\right)$.

The following proposition extends to the star-regular context some properties well known in the total and in the pointed contexts, and are needed for our work.

Proposition 2.6. In a star-regular category $\mathbb{C}$, the following conditions hold: 
(1) Consider a commutative diagram

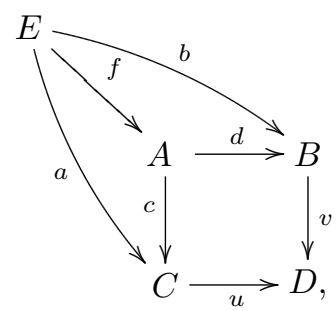

where $(c, d)$ is jointly monomorphic. Then

$$
E q(f)^{*}=E q(a)^{*} \wedge E q(b)^{*} .
$$

(2) Given a commutative diagram

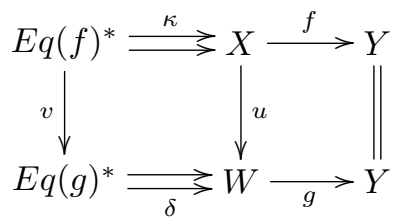

of stars and morphisms, the left-hand side diagram is a star-pullback.

(3) For a commutative diagram

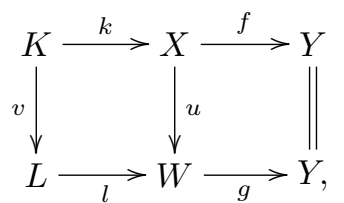

where $k$ and $l$ are the $\mathcal{N}$-kernels of $f$ and $g$, respectively, the left square is a pullback.

In order to prove the main result of this article, we need an additional assumption on the star-regular category. This property is referred to as having enough trivial objects in $[\mathbf{9}]$ (see also [12], and the references therein, for the related notion of a closed ideal of morphisms). There are several equivalent conditions defining when a category has enough trivial objects (see Proposition 3.5 in [9]). For the purpose of the present article, the following will be the most suitable one:

Definition $2.7([9])$. Let $(\mathbb{C}, \mathcal{N})$ be a regular multi-pointed category with $\mathcal{N}$-kernels. $\mathbb{C}$ has enough trivial objects when, for any relation $\left(r_{1}, r_{2}\right): R \rightrightarrows X$ in $\mathbb{C}$ and any arrow $f: K \rightarrow R$ such that $r_{1} f, r_{2} f \in \mathcal{N}$, one then has that $f \in \mathcal{N}$.

Example 2.8. It is clear that both in the total context and in the pointed context $\mathbb{C}$ has enough trivial objects. Besides the pointed and the total contexts, one can also consider the so-called proto-pointed context introduced in [10]: this is the situation of a regular multi-pointed category $(\mathbb{C}, \mathcal{N})$ with $\mathcal{N}$-kernels, where the class $\mathcal{N}$ consists of 
the morphisms $f: X \rightarrow Y$ whose regular image is the smallest subobject of $Y$. Recall also that a finitely complete category is quasi-pointed [3] if it has an initial object 0 , a terminal object 1 , and the unique arrow $0 \rightarrow 1$ is a monomorphism. As explained in [9] any quasi-pointed category provides an example of proto-pointed context: it suffices to choose for $\mathcal{N}$ the class of morphisms that factor through the initial object. Also, in the quasi-pointed context, $\mathbb{C}$ clearly has enough trivial objects.

Proposition 2.9. Let $\mathbb{C}$ be a star-regular category. If the following diagram

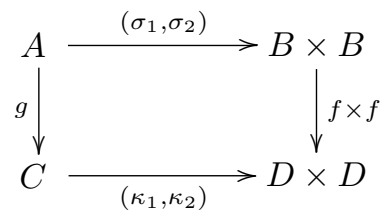

is a pullback with $\sigma_{1}, \kappa_{1} \in \mathcal{N}$, then the following commutative diagram

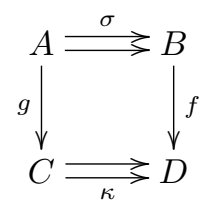

of stars and morphisms is a star-pullback. The converse is true when $f$ is a monomorphism and $\mathbb{C}$ has enough trivial objects.

Lemma 2.10. Let $\left(r_{1}, r_{2}\right): R \rightrightarrows X$ be a reflexive relation on an object $X$ in a starregular category $\mathbb{C}$, and let $\left(r_{1} k, r_{2} k\right): R^{*} \rightrightarrows X$ be the star associated with $R$. An arrow $q: X \rightarrow Y$ is the coequaliser of $\left(r_{1} k, r_{2} k\right)$ if and only if $q$ is the coequaliser of $\left(r_{1}, r_{2}\right)$.

Proof. In order to prove the result it suffices to show that an arrow $q: X \rightarrow Y$ coequalises $\left(r_{1}, r_{2}\right)$ whenever it coequalises $\left(r_{1} k, r_{2} k\right)$ :

$$
R^{*} \stackrel{k}{\longrightarrow} R \underset{r_{2}}{\stackrel{r_{1}}{\longrightarrow}} X \stackrel{q}{\longrightarrow} Y \text {. }
$$

Assume then that $q r_{1} k=q r_{2} k$, where $k$ is the $\mathcal{N}$-kernel of $r_{1}$. Since $r_{1}$ is a split epimorphism in a star regular category, $r_{1}$ is then the coequaliser of its kernel star $\left(\mu_{1}, \mu_{2}\right): E q\left(r_{1}\right)^{*} \rightrightarrows R$. Since $r_{1} \mu_{1}=r_{1} \mu_{2} \in \mathcal{N}$ there are unique morphisms $\theta_{i}$ such that $k \theta_{i}=\mu_{i}$ for $i \in\{1,2\}$ as in the diagram

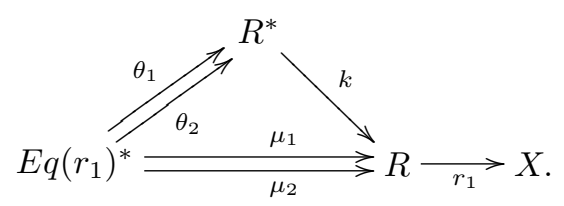


Consequently, we have

$$
\begin{aligned}
\left(q r_{2}\right) \mu_{1} & =q r_{2} k \theta_{1} \\
& =q r_{1} k \theta_{1} \quad\left(\text { since } q \text { coequalises }\left(r_{1} k, r_{2} k\right)\right) \\
& =q r_{1} \mu_{1} \\
& =q r_{1} \mu_{2} \quad\left(\text { since } r_{1} \text { coequalises }\left(\mu_{1}, \mu_{2}\right)\right) \\
& =q r_{1} k \theta_{2} \\
& =q r_{2} k \theta_{2} \\
& =\left(q r_{2}\right) \mu_{2} .
\end{aligned}
$$

It follows that there exists a unique $t: X \rightarrow Y$ such that $t r_{1}=q r_{2}$. Since the relation $R$ is reflexive, there is an arrow $\delta: X \rightarrow R$ such that $r_{1} \delta=1_{X}=r_{2} \delta$, and this implies that $t=t r_{1} \delta=q r_{2} \delta=q$, as desired.

Definition 2.11. A star-regular category $\mathbb{C}$ is said to be semi-effective star-regular when any equivalence relation $R \rightrightarrows X$ in $\mathbb{C}$ has the following property: if the star $R^{*}$ associated with $R$ is a subobject of a kernel star $E q(f)^{*}$

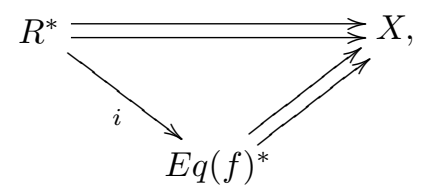

with $i$ a split monomorphism in $\mathbb{C}$, then $R^{*}$ is itself a kernel star.

Remark 2.12. If the category $\mathbb{C}$ has coequalisers of equivalence relations, then $\mathbb{C}$ is a semi-effective star-regular category if and only if for any equivalence relation $R \rightrightarrows X$ on an object $X$ and $q$ the coequaliser of the equivalence relation $R$

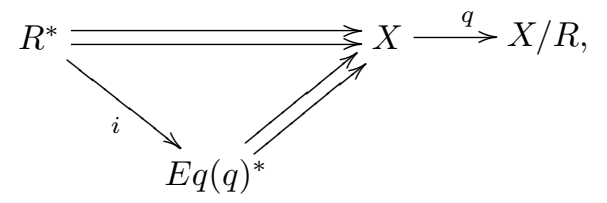

with $i$ a split monomorphism in $\mathbb{C}$, the star $R^{*}$ associated with $R$ is the kernel star of $q$, i.e., $R^{*} \cong E q(q)^{*}$.

Example 2.13. In the total context, it is obvious that any Barr-exact category is semieffective star-regular. More generally, any "efficiently regular category" in the sense of [4] is a semi-effective star-regular category, since any split monomorphism is a regular monomorphism. Examples of efficiently regular categories are provided by the category of topological groups and, more generally, by any category of topological models of a Mal'tsev algebraic theory (see Section 4.5).

In the pointed context, among the examples of semi-effective star-regular categories there are also any "category with a good theory of ideals" in the sense of [10], and any "almost abelian category" in the sense of $[\mathbf{2 4}]$. These examples, and many other ones, will be examined in Section 4. 


\section{Birkhoff subcategories}

By a regular-epireflective subcategory $\mathbb{D}$ of a regular category $\mathbb{C}$, we mean a full replete reflective subcategory

$$
\mathbb{C} \underset{U}{\stackrel{\perp}{\stackrel{\perp}{\rightleftarrows}}} \mathbb{D}
$$

with the property that the $A$-component $\eta_{A}: A \rightarrow U F(A)$ of the unit of the adjunction is a regular epimorphism for each $A \in \mathbb{C}$. It is well known that the last requirement is equivalent to the fact that $\mathbb{D}$ is closed in $\mathbb{C}$ under subobjects. $\mathbb{D}$ is a Birkhoff subcategory of $\mathbb{C}$ if, moreover, $\mathbb{D}$ is closed in $\mathbb{C}$ under regular quotients.

Lemma 2.14. Let $\mathbb{D}$ be a Birkhoff subcategory of a semi-effective star-regular category $\left(\mathbb{C}, \mathcal{N}_{\mathbb{C}}\right)$. Then $\mathbb{D}$ is a semi-effective star-regular category as well.

Proof. It is well known that if $\mathbb{C}$ is regular, then $\mathbb{D}$ is regular as well. This essentially follows from the fact that the regular epi-mono factorisation in $\mathbb{C}$ of an arrow in $\mathbb{D}$ is still the regular epi-mono factorisation of this arrow in $\mathbb{D}$. Since $\mathbb{D}$ is a full subcategory of $\mathbb{C}$, we can choose the ideal of morphisms of $\mathbb{D}$ induced by the ideal of morphisms in $\mathbb{C}$, so that, for any $X, Y$ in $\mathbb{D}, \mathcal{N}_{\mathbb{D}}(X, Y)=\mathcal{N}_{\mathbb{C}}(X, Y)=\mathcal{N}(X, Y)$. The fact that $\mathbb{D}$ is closed in $\mathbb{C}$ under subobjects implies that the $\mathcal{N}$-kernel of an arrow in $\mathbb{D}$ is computed in the same way in the categories $\mathbb{C}$ and $\mathbb{D}$.

The category $(\mathbb{D}, \mathcal{N})$ is a regular multi-pointed category. Since it is a regularepireflective subcategory of $\mathbb{C}$, any regular epimorphism in $\mathbb{D}$ is a regular epimorphism in $\mathbb{C}$, and $(\mathbb{D}, \mathcal{N})$ is a star-regular category.

Consider then the following diagram in $\mathbb{D}$ :

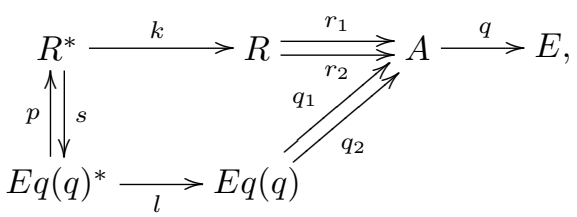

where $R \rightrightarrows A$ is an equivalence relation in $\mathbb{D}$ and $E q(q)^{*} \rightrightarrows A$ is a kernel star of its coequaliser $q$ (in $\mathbb{D}$ ), $k$ and $l$ are the $\mathcal{N}$-kernels of $r_{1}$ and $q_{1}$, respectively, and $s$ is a split monomorphism with $p s=1_{R^{*}}$, and $q_{i} l s=r_{i} k$ for $i \in\{1,2\}$. If we look at this diagram in $\mathbb{C}$, then $q$ is still the coequaliser of $\left(q_{1}, q_{2}\right)$ in $\mathbb{C}$, since $\mathbb{D}$ is stable in $\mathbb{C}$ under quotients. The category $\mathbb{C}$ is semi-effective star-regular, so that $\left(r_{1} k, r_{2} k\right): R^{*} \rightrightarrows A$ is a kernel star in $\mathbb{C}$ of its coequaliser $q^{\prime}: A \rightarrow E^{\prime}$ in $\mathbb{C}$. Moreover, $\mathbb{D}$ is stable in $\mathbb{C}$ under quotients, so that $E^{\prime}$ lies in $\mathbb{D}$, and $\left(r_{1} k, r_{2} k\right): R^{*} \rightrightarrows A$ is a kernel star in $\mathbb{D}$.

\section{Main result}

The following lemma will be needed to prove the main result of this article:

Lemma 3.1. Let $\mathbb{C}$ be a semi-effective star-regular category. Then, given a discrete fibration of equivalence relations 


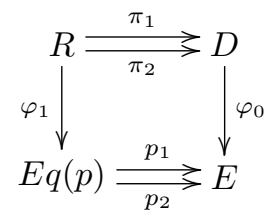

over the kernel pair of $p$, the coequaliser of $\pi_{1}$ and $\pi_{2}$ exists.

Proof. Let us build the following diagram:

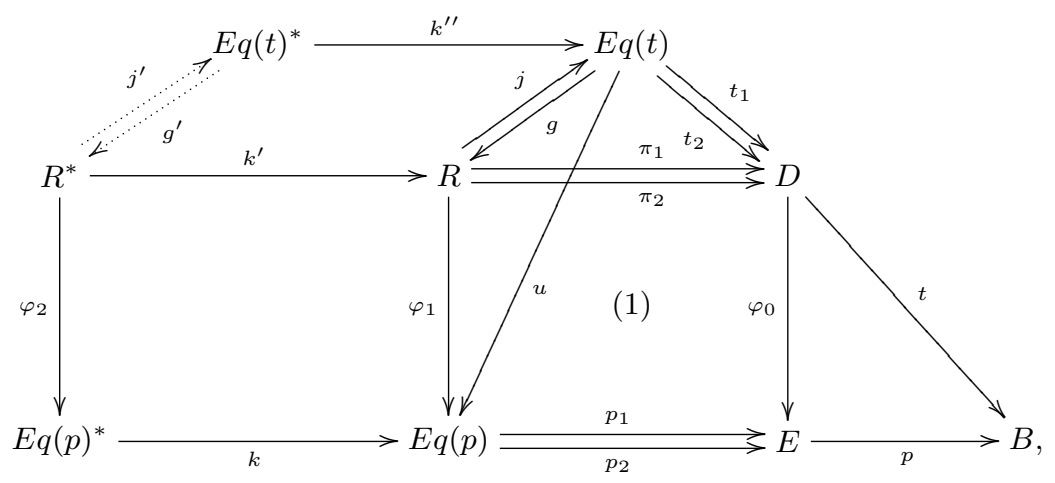

where

(a) $t=p \varphi_{0}$;

(b) the morphisms $k, k^{\prime}$ and $k^{\prime \prime}$ are the $\mathcal{N}$-kernels of $p_{1}, \pi_{1}$ and $t_{1}$, respectively;

(c) $u$ is the unique arrow such that $\varphi_{0} t_{l}=p_{l} u$, for $l \in\{1 ; 2\}$;

(d) $g$ is the unique arrow such that $\pi_{1} g=t_{1}$ and $\varphi_{1} g=u$.

There is a unique morphism $j$ such that $t_{l} j=\pi_{l}$, for $l \in\{1 ; 2\}$. Since $\left(\varphi_{1}, \pi_{1}\right)$ is jointly monomorphic and $u j=\varphi_{1}$, we have the equality $g j=1_{R}$. By assumption $R^{*} \rightrightarrows D$ is then a kernel star, since $E q(t)^{*} \rightrightarrows D$ is a kernel star, and the induced arrow $j^{\prime}$ a split monomorphism. This implies that the coequaliser $q: D \rightarrow D / R^{*}$ of $\pi_{1} k^{\prime}$ and $\pi_{2} k^{\prime}$ exists, and $q$ is also the coequaliser of $\pi_{1}$ and $\pi_{2}$ by Lemma 2.10 .

Theorem 3.2. Let $\mathbb{C}$ be a semi-effective star-regular category with enough trivial objects. Then the following conditions for an arrow $p: E \rightarrow B$ are equivalent:

(1) $p$ is an effective descent morphism;

(2) $p$ is a descent morphism;

(3) $p$ is a regular epimorphism.

Proof. The implication (1) $\Rightarrow(2)$ is trivial, whereas $(2) \Rightarrow(3))$ is true in any finitely complete regular category (see Theorem 1.1).

We are now going to prove that $(3) \Rightarrow(1)$. Assume that $p: E \rightarrow B$ is a regular epimorphism, and consider the commutative diagram (1) as in Lemma 3.1. We would like to prove that the equivalence relation $R \rightrightarrows D$ is effective. 
Let us then build the commutative diagram

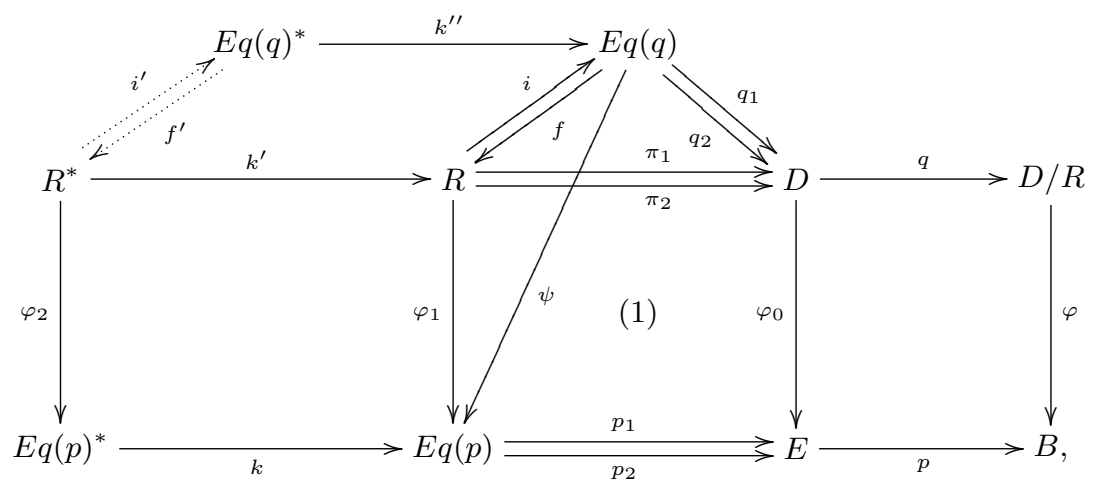

where

(a) $q$ is the coequaliser of $\pi_{1}$ and $\pi_{2}$ (which exists by Lemma 3.1);

(b) $i$ is the unique arrow such that $q_{h} i=\pi_{h}$, for $h \in\{1 ; 2\}$;

(c) $k, k^{\prime}$ and $k^{\prime \prime}$ are the $\mathcal{N}$-kernels of $p_{1}, \pi_{1}$ and $q_{1}$, respectively;

(d) $\psi$ is the unique arrow such that $\varphi_{0} q_{h}=p_{h} \psi$, for $h \in\{1 ; 2\}$;

(e) $f$ is the unique arrow such that $\pi_{1} f=q_{1}$ and $\varphi_{1} f=\psi$.

A similar argument to the one used in Lemma 3.1 (to show that $g j=1_{R}$ ) implies that $f i=1_{R}$. We also know that the induced split monomorphism $i^{\prime}: R^{*} \rightarrow E q(q)^{*}$ is an isomorphism, thanks to Lemma 3.1 and to Remark 2.12.

It will suffice to show that the split epimorphism $f: E q(q) \rightarrow R$ is a monomorphism, and this will imply that $i: R \rightarrow E q(q)$ will be an isomorphism, as desired.

For this consider the following commutative cube:

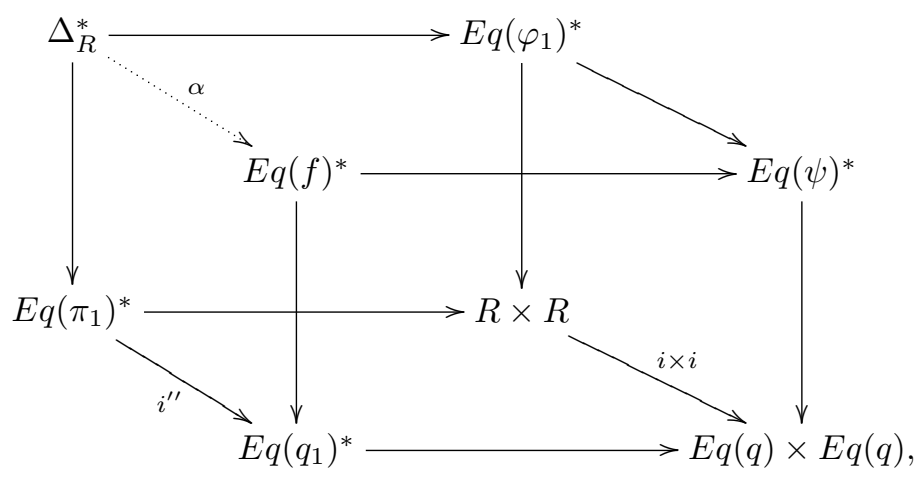

where the back square is a pullback by Corollary 2.5 , the front square is a pullback by Proposition 2.6(1), and the dotted arrow $\alpha$ is induced by the universal property of this latter pullback. By Proposition 2.6(2), the commutative diagram

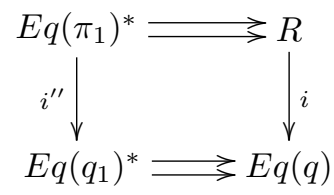


is a star-pullback, and by Proposition 2.9 the bottom square of the cube above is a pullback. The same arguments are applied to conclude that the right square is a pullback as well, so that the left square is a pullback. The arrow $i^{\prime \prime}$ is an isomorphism: indeed, this follows from Proposition 2.6(2) and (3), the fact that $i^{\prime}: R^{*} \rightarrow E q(q)^{*}$ is an isomorphism, and the assumption that $\mathbb{C}$ has enough trivial objects. Accordingly, the dotted arrow $\alpha$ is an isomorphism as well. This implies that the projections $f_{1}: E q(f)^{*} \rightarrow E q(q)$ and $f_{2}: E q(f)^{*} \rightarrow E q(q)$ of the kernel star of $f$ are equal, as one can see from the commutativity of the following diagram:

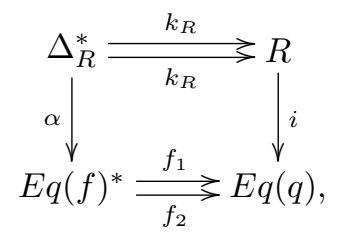

where $k_{R}$ is the $\mathcal{N}$-kernel of $1_{R}$. From Lemma 2.4(c) we conclude that $f$ is a monomorphism, as desired.

\section{Examples}

\subsection{Categories with a good theory of ideals}

In any regular multi-pointed category $\mathbb{C}$ there is a natural notion of ideal, that extends the one coming from universal algebra considered in $[\mathbf{2 1}, \mathbf{2 5}$. A star-relation $\left(r_{1}, r_{2}\right): R \rightrightarrows X$ in $\mathbb{C}$ is an ideal if it is the regular image of a kernel star: this means that there exists a kernel star $\left(k_{1}, k_{2}\right): K \rightrightarrows Y$ and a regular epi $f: Y \rightarrow X$ with the property that $f(K)=R$. This categorical notion of ideal, introduced in the context of regular multi-pointed categories in [10], extends the one defined and studied in regular categories in $[\mathbf{1 4}, \mathbf{1 5}]$. It is obvious that any kernel star is an ideal, but the converse is not true, in general, even for varieties of algebras. When the classes of ideals and of kernel stars coincide in a star-regular category $\mathbb{C}$, one says that $\mathbb{C}$ is a category with a good theory of ideals [10]. The results in [10] show that, in the total context, the categories with a good theory of ideals are exactly the exact Goursat categories $[\mathbf{6}]$, whereas, in the pointed context, they are the so-called ideal determined categories introduced in [13] (in the presence of finite colimits as required in [13]).

Let us then observe that, in the pointed context, the star $\left(r_{1} k, r_{2} k\right): R^{*} \rightrightarrows X$ associated with an equivalence relation $\left(r_{1}, r_{2}\right): R \rightrightarrows X$ on $X$ is the star whose first component is the zero arrow, and the second one the normal subobject in the sense of Bourn [3] associated with $R$, i.e., the 0 -class of $R$.

In any category $\mathbb{C}$ with a good theory of ideals, it is possible to show that any star $\left(r_{1} k, r_{2} k\right): R^{*} \rightrightarrows X$ associated with an equivalence relation $\left(r_{1}, r_{2}\right): R \rightrightarrows X$ on $X$ is necessarily a kernel star. Indeed, any such equivalence relation determines a canonical discrete fibration $\left(r_{2}, \sigma\right): E q\left(r_{1}\right) \rightarrow R$ as in the diagram

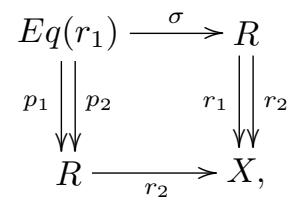


where $\sigma$ is the arrow (internally) sending an element $((x, y),(x, z))$ of $E q\left(r_{1}\right)$ to the element $(y, z)$ of $R$. The following pushout

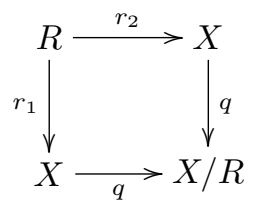

exists in $\mathbb{C}$, since $r_{1}$ and $r_{2}$ are regular epimorphisms (see [10]). Thanks to the characterisation of the categories with a good theory of ideals given in Theorem 3.8 in [10], it follows that the induced composite arrow $E q\left(r_{1}\right)^{*} \rightarrow R^{*} \rightarrow E q(q)^{*}$ is a regular epi. This implies that the canonical monomorphism $R^{*} \rightarrow E q(q)^{*}$ is also a regular epi, thus an isomorphism.

Accordingly, any category $\mathbb{C}$ with a good theory of ideals is semi-effective starregular. By Theorem 3.2 , when $\mathbb{C}$ also has enough trivial objects, any regular epimorphism in $\mathbb{C}$ is then an effective descent morphism.

The particular case of ideal determined categories has been considered by T. Everaert [8], who arrived at the conclusion that regular epimorphisms therein are effective for descent by using a completely different approach. Observe that our Theorem 3.2 applies to any star-regular category with enough trivial objects for which the class of "stars of equivalence relations" coincides with the class of "kernels stars". This latter condition is weaker than the one asserting that the class of "ideals" coincide with the class of "kernel stars".

The property of being a category with a good theory of ideals is stable under Birkhoff subcategories. More precisely, one has the following

Proposition 4.1. Let $\mathbb{D}$ be a regular-epireflective subcategory of a category $\mathbb{C}$ with a good theory of ideals. Then the following conditions are equivalent:

(1) $\mathbb{D}$ is a Birkhoff subcategory of $\mathbb{C}$;

(2) $\mathbb{D}$ is a category with a good theory of ideals;

(3) For any span of regular epimorphisms $C \stackrel{g}{\longleftarrow} A \stackrel{f}{\longrightarrow} B$ in $\mathbb{D}$ their pushout $\left(P, f^{\prime}, g^{\prime}\right)$ in $\mathbb{C}$ is also their pushout in $\mathbb{D}$.

Proof of (1) $\Rightarrow$ (2). By Lemma 2.14 we know that the category $\mathbb{D}$ is star-regular. Consider then the diagram

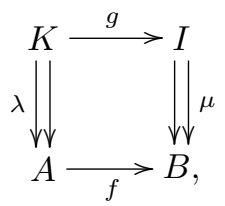

where $f$ is a regular epimorphism in $\mathbb{D}, \lambda$ a kernel star in $\mathbb{D}$ and $\mu g$ the factorisation (regular epi)-(monic-star) of $f \lambda$ in $\mathbb{C}$. One clearly has that $I \in \mathbb{D}$. By assumption $\mu$ is then the kernel star of its coequaliser $q: B \rightarrow Q$ in $\mathbb{C}$. The category $\mathbb{D}$ is stable in $\mathbb{C}$ under quotients, and this implies that $q: B \rightarrow Q$ is also the coequaliser of $\mu$ in $\mathbb{D}$. Accordingly, $\mu$ is a kernel star in $\mathbb{D}$. 
(2) $\Rightarrow$ (3). Let $f: A \rightarrow B$ and $g: A \rightarrow C$ be regular epimorphisms lying in $\mathbb{D}$. In the category $\mathbb{C}$ with a good theory of ideals the pushout square

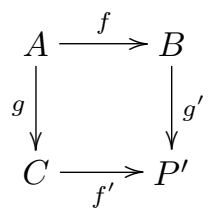

always exists: it is obtained by considering the coequaliser $g^{\prime}: B \rightarrow P^{\prime}$ of the regular image $f\left(E q(g)^{*}\right) \rightrightarrows B$ of the kernel star $E q(g)^{*} \rightrightarrows A$ along $f$ (by Theorem 3.8 in $[\mathbf{1 0}])$. The assumption that $\mathbb{D}$ is a category with a good theory of ideals implies that the pushout $\left(P^{\prime \prime}, f^{\prime \prime}, g^{\prime \prime}\right)$ of $f$ and $g$ exists also in $\mathbb{D}$, and $g^{\prime \prime}$ is the coequaliser of $f\left(E q(g)^{*}\right) \rightrightarrows B$ in $\mathbb{D}$. The canonical comparison $\eta: P^{\prime} \rightarrow P^{\prime \prime}$ such that $\eta g^{\prime}=g^{\prime \prime}$ is a regular epimorphism. The fact that both $g^{\prime}$ and $g^{\prime \prime}$ are regular epimorphisms in $\mathbb{C}$ with the same kernel star implies that the arrow $\eta: P^{\prime} \rightarrow P^{\prime \prime}$ is an isomorphism.

(3) $\Rightarrow$ (1). Let $f: A \rightarrow B$ be a regular epimorphism in $\mathbb{C}$, with $A \in \mathbb{D}$, and consider the kernel pair $\left(f_{1}, f_{2}\right): E q(f) \rightrightarrows A$ of $f$, which lies in $\mathbb{D}$, since $\mathbb{D}$ is stable in $\mathbb{C}$ under subobjects. The projections $f_{1}: E q(f) \rightarrow A$ and $f_{2}: E q(f) \rightarrow A$ are regular epimorphisms in $\mathbb{D}$, so that $(B, f, f)$ is their pushout in $\mathbb{D}$ by the assumption. This shows that $B \in \mathbb{D}$.

Observe that Proposition 4.1 is useful to find examples of semi-effective star-regular categories which are not categories with a good theory of ideals.

This is the case, for instance, for the category $\mathrm{Ab}_{t . f}$. of torsion-free abelian groups. Indeed, $A b_{t . f .}$. is obviously a normal category, and it is also semi-effective star-regular (see Example 4.4); however, by Proposition 4.1, it does not have a good theory of ideals. This is due to the fact that, although $A b_{t . f}$. is a regular-epireflective subcategory of the category $A b$ of abelian groups, it is not stable in $A b$ under quotients.

\subsection{Regular epimorphisms in a category with a good theory of ideals}

When $\mathbb{C}$ is a category, we denote by $\operatorname{RegEpi}(\mathbb{C})$ the category of regular epimorphisms in $\mathbb{C}$ : an object in RegEpi(C) is a regular epi $a: A_{1} \rightarrow A_{0}$ in $\mathbb{C}$, and a morphism $f: a \rightarrow b$ in RegEpi $(\mathbb{C})$ is a pair $\left(f_{0}, f_{1}\right)$ of morphisms in $\mathbb{C}$ with $f_{0}: A_{0} \rightarrow B_{0}$ and $f_{1}: A_{1} \rightarrow B_{1}$ such that $f_{0} a=b f_{1}$.

Lemma 4.2. Let $\mathbb{C}$ be a category with a good theory of ideals and enough trivial objects. Then RegEpi($(\mathbb{C})$ is star-regular with enough trivial objects.

Proof. The category RegEpi $(\mathbb{C})$ is finitely complete, and it also has coequalisers of effective equivalence relations since $\mathbb{C}$ has pushouts of regular epimorphisms by regular epimorphisms (see also $[\mathbf{8}]$ ). Furthermore, a regular epi in RegEpi $(\mathbb{C})$ is simply given by a pair of regular epimorphisms $\left(f_{0}, f_{1}\right): a \rightarrow b$ determining a pushout in $\mathbb{C}$. The assumption that $\mathbb{C}$ has enough trivial objects and the characterisation of the categories with a good theory of ideals given in Theorem 3.8 in [10] allow one to prove that regular epimorphisms in $\operatorname{RegEpi}(\mathbb{C})$ are stable under pullbacks, and $\operatorname{RegEpi}(\mathbb{C})$ is then a regular category. Let us then denote by $\mathcal{M}$ the class of morphisms $f=\left(f_{0}, f_{1}\right)$ in RegEpi($(\mathbb{C})$ defined by $f=\left(f_{0}, f_{1}\right) \in \mathcal{M}$ if and only if $f_{1} \in \mathcal{N}$. This class $\mathcal{M}$ is an ideal of morphisms in $\operatorname{RegEpi}(\mathbb{C})$ (since $\mathcal{N}$ is an ideal in $\mathbb{C}$ ), so that $(\operatorname{RegEpi}(\mathbb{C}), \mathcal{M})$ 
is a regular multi-pointed category. The $\mathcal{M}$-kernel of a morphism $f=\left(f_{0}, f_{1}\right): a \rightarrow b$ in RegEpi $(\mathbb{C})$ is constructed as follows: one takes the $\mathcal{N}$-kernel $k_{1}$ of $f_{1}$, and then the factorisation $k_{0} c$ of $a k_{1}$ as a regular epimorphism $c$ followed by a monomorphism $k_{0}$. The arrow $\left(k_{0}, k_{1}\right)$ is the $\mathcal{M}$-kernel of $f$ in $\operatorname{RegEpi}(\mathbb{C})$. Since any morphism in $(\mathbb{C}, \mathcal{N})$ has an $\mathcal{N}$-kernel by assumption, then any morphism in RegEpi(C) has an $\mathcal{M}$-kernel.

To see that any regular epimorphism in $\operatorname{RegEpi(}(\mathbb{C})$ is the coequaliser of its kernel star, consider a regular epimorphism $\left(e_{0}, e_{1}\right): a \rightarrow b$ in RegEpi(C). The induced arrow $c: E q\left(e_{1}\right)^{*} \rightarrow E q\left(e_{0}\right)^{*}$ is a regular epimorphism (again by Theorem 3.8 in [10]). If $\left(\left(m_{1}, \kappa_{1}\right),\left(m_{2}, \kappa_{2}\right)\right): c \rightrightarrows a$ is the kernel star of $\left(e_{0}, e_{1}\right)$, then $\left(e_{0}, e_{1}\right)$ is the coequaliser of $\left(\left(m_{1}, \kappa_{1}\right),\left(m_{2}, \kappa_{2}\right)\right): c \rightrightarrows a$, and RegEpi(C) is a star-regular category. If $\mathbb{C}$ has

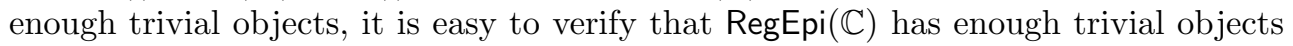
as well.

Remark 4.3. It is not true, in general, that RegEpi(C) has a good theory of ideals when $\mathbb{C}$ is a category with a good theory of ideals (and enough trivial objects). For instance, in the total context, even when $\mathbb{C}$ is abelian, the category RegEpi($(\mathbb{C})$ is regular but not exact Goursat (= with a good theory of ideals, in the total context).

From Lemma 4.2, and Corollary 2.4 in [8], we get

Corollary 4.4. Let $\mathbb{C}$ be a category with a good theory of ideals and enough trivial objects. Then regular epimorphisms are effective descent morphisms in RegEpi($(\mathbb{C})$.

Under the assumptions of Corollary 4.4 it can be shown that, more generally, the categories RegEpi $(\mathbb{C})^{n}$ of $n$-fold regular epimorphisms in $\mathbb{C}$ are star-regular with enough trivial objects (see also Proposition 3.1 in $[\mathbf{8}]$ ).

\subsection{Monomorphisms in a semi-effective star-regular category}

Let us denote by Mono $(\mathbb{C})$ the category of monomorphisms in a category $\mathbb{C}$. An object in Mono( $\mathbb{C})$ is a monomorphism $m: M_{1} \rightarrow M_{0}$ in $\mathbb{C}$ and a morphism $f: m \rightarrow n$ in Mono $(\mathbb{C})$ is a pair $\left(f_{0}, f_{1}\right)$ of morphisms of $\mathbb{C}$ with $f_{0}: M_{0} \rightarrow N_{0}$ and $f_{1}: M_{1} \rightarrow N_{1}$ such that $f_{0} m=n f_{1}$. When $(\mathbb{C}, \mathcal{N})$ is a regular multi-pointed category, let us denote by $\mathcal{M}$ the class of morphisms $k=\left(k_{0}, k_{1}\right)$ of $\operatorname{Mono}(\mathbb{C})$ such that $k_{0}$ and $k_{1}$ are in $\mathcal{N}$. In this way, $(\operatorname{Mono}(\mathbb{C}), \mathcal{M})$ becomes a regular multi-pointed category as well. The reader will find it easy to verify the following

Lemma 4.5. Let $(\mathbb{C}, \mathcal{N})$ be a semi-effective star-regular category with enough trivial objects. Then $\operatorname{Mono}(\mathbb{C})$ is a semi-effective star-regular category with enough trivial objects.

As a consequence, under the assumptions of the lemma above, the regular epimorphisms are effective descent morphisms in $\operatorname{Mono}(\mathbb{C})$ (see also Example 4.4 in $[8]$ ).

\subsection{Almost abelian categories}

Another class of examples to which Theorem 3.2 applies is provided by the socalled "almost abelian categories" in the sense of W. Rump [24], also called "Raikov semi-abelian" $[\mathbf{2 2}]$ in the literature. An almost abelian category can be defined as an additive category with kernels and cokernels with the property that normal epimorphisms are pullback-stable and normal monomorphisms are pushout-stable. As 
explained in $[\mathbf{2 3}]$, G. Janelidze has observed that a category $\mathbb{C}$ is almost abelian if and only if it is both homological (in the sense of F. Borceux and D. Bourn [2]) and co-homological. It is well known that, in an almost abelian category, any arrow $f: A \rightarrow B$ has a canonical factorisation

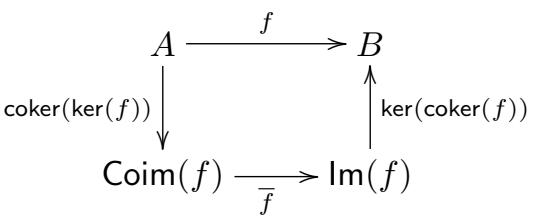

with $\bar{f}$ : $\operatorname{Coim}(f) \rightarrow \operatorname{Im}(f)$ a bimorphism, i.e., an arrow which is at the same time monic and epic. Any almost abelian category $\mathbb{C}$ is normal (it is even homological), and we are now going to prove that it is also semi-effective star-regular (with respect to the class $\mathcal{N}$ of zero arrows). For this, consider a commutative diagram

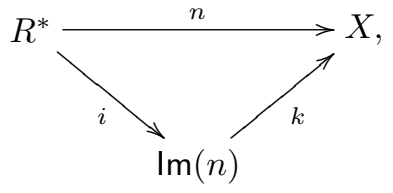

where $n: R^{*} \rightarrow X$ is the (Bourn-)normal monomorphism yielding the 0-class of an equivalence relation $R, k=\operatorname{ker}(\operatorname{coker}(n))$ is the kernel of the cokernel coker $(n)$ of $n$, and $i$ is a split monomorphism. Then, by factorising $n=k \bar{n} \operatorname{coker}(\operatorname{ker}(n))$ as in diagram (2), we see that the arrow $i=\operatorname{coker}(\operatorname{ker}(n)) \bar{n}$ is an epimorphism, as a composite of two epimorphisms. It follows that $i$ is an isomorphism, and $\mathbb{C}$ is a semi-effective star-regular category, as desired.

We observe that, more generally, any normal category $\mathbb{C}$ such that any arrow in $\mathbb{C}$ has a factorisation as an epimorphism followed by a normal monomorphism is semi-effective star-regular.

It is explained in $[\mathbf{2 4}]$ that any torsion-free subcategory of an abelian category $\mathbb{C}$ is necessarily almost abelian, as is any torsion subcategory of $\mathbb{C}$. Further examples of almost abelian categories are given, for instance, by the categories of real (or complex) normed vector spaces, Banach spaces (with bounded linear maps as morphisms), and also by the category of locally compact abelian groups. By Theorem 3.2 the regular epimorphisms are then effective descent morphisms in all these categories.

\subsection{Categories of topological Mal'tsev algebras}

Consider $\mathbb{T}$ a Mal'tsev theory, i.e., an algebraic theory containing a ternary term $p(x, y, z)$ satisfying the identities $p(x, x, y)=y$ and $p(x, y, y)=x$. The category $\mathbb{T}$ (Top) of topological models of such a theory (i.e., models in the category Top of topological spaces) is called a category of topological Mal'tsev algebras. The category $\mathbb{T}$ (Top) is a regular category, as shown in $[\mathbf{2 0}]$. We now prove that $\mathbb{T}($ Top $)$ is semieffective star-regular (thinking of $(\mathbb{T}($ Top $), \mathcal{N})$ as a star-regular category with $\mathcal{N}$ the ideal of all morphisms).

When $\left(R, \tau_{R}\right) \rightrightarrows\left(X, \tau_{X}\right)$ is an equivalence relation in $\mathbb{T}($ Top $)$, and we consider a commutative diagram 


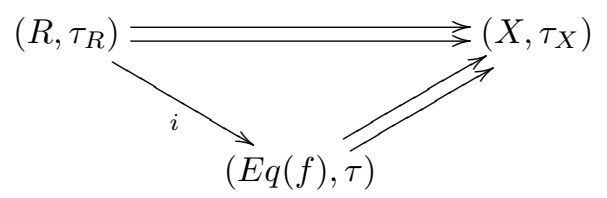

with the property that $i$ is a split monomorphism, then the topology of $\left(R, \tau_{R}\right)$ is the one induced by the topology of the product $\left(X \times X, \tau_{X \times X}\right)$. Accordingly, the equivalence relation $\left(R, \tau_{R}\right)$ is the kernel pair of its coequaliser. Hence, the category $\mathbb{T}$ (Top) is semi-effective star-regular and, by Theorem 3.2, every regular epimorphism is an effective descent morphism in $\mathbb{T}$ (Top) (this result is known, see [11] for instance, although the proof presented here is different).

\section{References}

[1] M. Barr, P.A. Grillet and D.H. van Osdol, Exact categories and categories of sheaves, Lecture Notes in Mathematics 236, Springer-Verlag, New York, 1971.

[2] F. Borceux and D. Bourn, Mal'cev, protomodular, homological and semiabelian categories, Homological and semi-abelian categories, Mathematics and its Applications 566, Kluwer Academic Publishers, Dordrecht (2004).

[3] D. Bourn, Normal subobjects and abelian objects in protomodular categories, J. Algebra 228 (2000), no. 1, 143-164.

[4] D. Bourn, Baer sums in homological categories, J. Algebra 308 (2007), no. 1, 414-443.

[5] D. Bourn and Z. Janelidze, Pointed protomodularity via natural imaginary subtractions, J. Pure Appl. Algebra 213 (2009), no. 9, 1835-1851.

[6] A. Carboni, G.M. Kelly, and M.C. Pedicchio, Some remarks on Mal'tsev and Goursat categories, Appl. Cat. Struct. 1 (1993), no. 4, 385-421.

[7] C. Ehresmann, Sur une notion générale de cohomologie, C. R. Acad. Sci. Paris 259 (1964) 2050-2053.

[8] T. Everaert, Effective descent morphisms of regular epimorphisms, J. Pure Appl. Algebra 216 (2012), no. 8-9, 1896-1904.

[9] M. Gran, Z. Janelidze and D. Rodelo, $3 \times 3$ lemma for star-exact sequences, Homology, Homotopy Appl. 14, no. 2 (2012), 1-22.

[10] M. Gran, Z. Janelidze and A. Ursini, A good theory of ideals in regular multipointed categories, J. Pure Appl. Algebra 216 (2012), no. 8-9, 1905-1919.

[11] M. Gran and V. Rossi, Torsion theories and Galois coverings of topological groups, J. Pure Appl. Algebra 208 (2007), no. 1, 135-151.

[12] M. Grandis, On the categorical foundations of homological and homotopical algebra, Cah. Top. Géom. Diff. Catég. 33 (1992), no. 2, 135-175.

[13] G. Janelidze, L. Márki, W. Tholen and A. Ursini, Ideal determined categories, Cah. Top. Géom. Diff. Catég. 51 (2010), no. 2, 115-125.

[14] G. Janelidze, L. Márki and A. Ursini, Ideals and clots in universal algebra and in semi-abelian categories, J. Algebra 307 (2007), no. 1, 191-208. 
[15] G. Janelidze, L. Márki and A. Ursini, Ideals and clots in pointed regular categories, Appl. Categ. Struct. 17 (2009), no. 4, 345-350.

[16] G. Janelidze and M. Sobral, Descent for regular epimorphisms in Barr exact Goursat categories, Appl. Categ. Struct. 19 (2011), no. 1, 271-276.

[17] G. Janelidze, M. Sobral and W. Tholen, Beyond Barr exactness: effective descent morphisms, in Categorical Foundations: Special Topics in Order, Topology, Algebra and Sheaf Theory (M.C. Pedicchio and W. Tholen, eds.), Encycl. of Math. Appl. 97, Cambridge University Press, Cambridge (2004), 359-405.

[18] G. Janelidze and W. Tholen, Facets of descent, I, Appl. Categ. Struct. 2 (1994), no. $3,245-281$.

[19] Z. Janelidze, The pointed subobject functor, $3 \times 3$ lemmas and subtractivity of spans, Th. Appl. Categ. 23 (2010), no. 11, 221-242.

[20] P.T. Johnstone and M.C. Pedicchio, Remarks on continuous Mal'cev algebras, Rend. Istit. Mat. Univ. Trieste 25 (1993), no. 1-2, 277-297.

[21] R. Magari, Su una classe equazionale di algebre, Ann. Mat. Pura Appl. (4) 75 (1967), 277-311.

[22] D.A. Raikov, Semiabelian categories, Soviet Math. Doklady 10 (1969), 12421245.

[23] J. Rosický and W. Tholen, Factorization, fibration and torsion, J. Homotopy Relat. Struct. 2 (2007), no. 2, 295-314.

[24] W. Rump, Almost abelian categories, Cah. Topol. Géom. Diifér. Catégor. 42 (2001), no. 3, 163-225.

[25] A. Ursini, Sulle varietá di algebre con una buona teoria degli ideali, Boll. Unione Mat. Ital. (4) 6 (1972), 90-95.

[26] A. Ursini, On subtractive varieties. I, Algebra Univers. 31 (1994), no. 2, 204222 .

Marino Gran marino.gran@uclouvain.be

Institut de Recherche en Mathématique et Physique, Université catholique de Louvain, Chemin du Cyclotron 2, 1348 Louvain-la-Neuve, Belgium

Olivette Ngaha Ngaha mathilde.ngahangaha@uclouvain.be

Institut de Recherche en Mathématique et Physique, Université catholique de Louvain, Chemin du Cyclotron 2, 1348 Louvain-la-Neuve, Belgium 